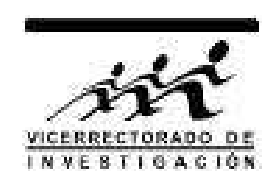

\title{
Avances en la caracterización mineralógica de los suelos del río Chancay, Lambayeque, por difractometría de rayos $\mathrm{X}$ y espectroscopia Mössbauer
}

\author{
Alejandro L. Trujillo Quinde*1, Miriam E. Mejía Santillan${ }^{1}$, Jorge A. Bravo Cabrejos ${ }^{1}$, María L. Cerón \\ Loayza $^{1}$ y Eleazar Rufasto ${ }^{2}$ \\ ${ }^{1}$ Laboratorio de Análisis de Suelos, Facultad de Ciencias Físicas, Universidad Nacional Mayor de San \\ Marcos, Lima, Perú \\ ${ }^{2}$ Facultad de Agronomía, Universidad Nacional Pedro Ruiz Gallo, Lambayeque, Perú
}

Recibido 20 marzo 2011 - Aceptado 30 diciembre 2011

\begin{abstract}
El presente trabajo reporta los resultados preliminares de la caracterización mineralógica de los suelos del Valle del Río Chancay, Lambayeque, mediante las técnicas de difractometría de rayos X (DRX) y espectroscopia Mössbauer por transmisión (EMT). La muestra de estudio, SI53, proviene de uno de los dos horizontes de de un perfil de suelo de la localidad de Sialupe localizada en el Valle del Río Chancay . La DRX permitió la identificación de las fases mineralógicas cristalinas en la muestra SI53 en bruto a $30 \mathrm{~cm}$ de profundidad, tales como cuarzo, montmorillonita, clinocloro, muscovita, anfibol y albita; estos mismos minerales, excepto el anfibol, se aprecian también en la correspondiente fracción arcillosa. La EMT permitió identificar, en la muestra en bruto, un sexteto adjudicado a la hematita y un doblete central que podría ser adjudicado a un óxido de hierro en estado superparamagnético o a la caolinita; los otros dobletes son adjudicados a la montmorillonita, la moscovita y la epidota. Todas estas fases, excepto la epidota, también son identificadas por la EMT en la fracción arcillosa.

Palabras claves: Suelo, fracción arcillosa, difractometría de rayos X, espectroscopia Mössbauer, estado superparamagnético.
\end{abstract}

\section{Advances in the mineralogical characterization of soils from the Chancay River, Lambayeque, by X-ray difraction and Mössbauer spectroscopy}

This paper reports the preliminary results of the mineralogical characterization of soils from the Chancay River Valley, Lambayeque, by X ray diffractometry (XRD) and transmission Mössbauer spectroscopy (TMS). The studied soil sample, SI53, belongs to one of the two horizons of a selected soil profile from the area of Sialupe, located in the Chancay River Valley. The XRD allowed the identification of crystalline mineralogical phases in the such as found sample, at a depth of $30 \mathrm{~cm}$, such as quartz, montmorillonite, clinochlore, amphibole and albite; these same minerals, except amphibole, appear also in its corresponding clay fraction. The TMS allowed the identification, in the such as found sample, of a sextet assigned to hematite and a central doublet that can be assigned to an iron oxide in the super paramagnetic state or to kaolinite; the other doublets are assigned to montmorillonite, muscovite and epidote. All of these phases, with the exception of epidote, are also identified in the clay fraction by TMS.

Keywords: Soil, clay fraction, X ray diffractometry, Mössbauer spectroscopy, superparamagnetic state.

La muestra en estudio proviene de uno de los dos horizontes seleccionados de una calicata ubicada en la localidad de Sialupe, Valle del Río Chancay, Lambayeque. Este trabajo se inició con el análisis de una muestra proveniente del punto 53, que corresponde a un suelo cultivado de la región de Sialupe. La posición del punto de muestreo corresponde a las coordenadas SAD 56: $X=625672 \mathrm{~m} Y=9264850 \mathrm{~m}$; WGS84: $X=625414$ m $Y=9264482 \mathrm{~m}$ (figura 1). Los cultivos de mayor preponderancia en la zona son el arroz y el algodón, y

*atrujilloq@unmsm.edu.pe 
en menor escala maíz amarillo, caña de azúcar y pastos. El cultivo de frutales es casi insignificante. Este trabajo se viene llevando a cabo bajo la cooperación entre el Laboratorio de Análisis de Suelos de la Facultad de Ciencias Físicas de la Universidad Nacional Mayor de San Marcos y la Facultad de Agronomía de la Universidad Nacional Pedro Ruiz gallo de Lambayeque.

\section{Materiales y Métodos}

Se hicieron calicatas obteniéndose muestras a superficie, y a $30 \mathrm{~cm}$ de profundidad. Las cuales fueron codificadas como SI para la región de Sialupe y el número correspondiente al punto de extracción. Se analizó la muestra SI53 a $30 \mathrm{~cm}$ de profundidad, tanto en bruto como en su fracción arcillosa.

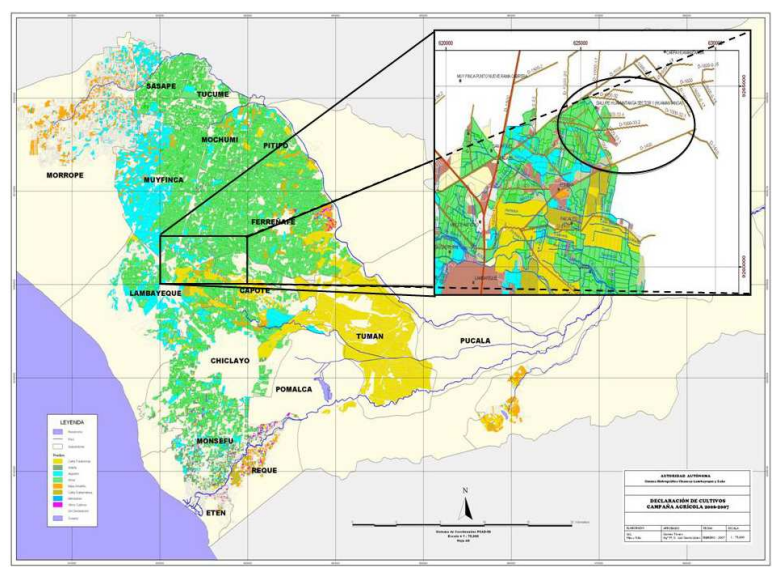

Figura 1: Ubicación de la zona de estudio.

\section{Preparación de las muestras}

Las muestras en bruto fueron secadas al aire libre y luego tamizadas en malla de $2 \mathrm{~mm}$. También se obtuvo la fracción arcillosa por sedimentación (figura 2). Para analizar las muestras en bruto y la fracción arcilla por EMT y DRX estas fueron molidas con un mortero de ágata con el propósito de obtener una muestra en polvo de tamaño de grano suficientemente fino [1, la cual se colocó en los portamuestras respectivos. Todo este protocolo se esquematiza en la figura 3 .

\section{Difracción de rayos $\mathrm{X}$}

Se empleó un difractómetro marca RIGAKU, modelo MINIFLEX, utilizando radiación de $\mathrm{Cu}-\mathrm{K}_{\alpha},(30 \mathrm{kV}$, $15 \mathrm{~mA}$ ) en un goniómetro vertical y con monocromador. Los difractogramas cubrieron el rango entre $15^{\circ}$ y $90^{\circ}$ usando un paso $2 \theta$ de $0.02^{\circ}$ y un tiempo de conteo de 2 segundos por cada incremento de $2 \theta$. La cantidad de muestra utilizada fue de $2 \mathrm{~g}$.

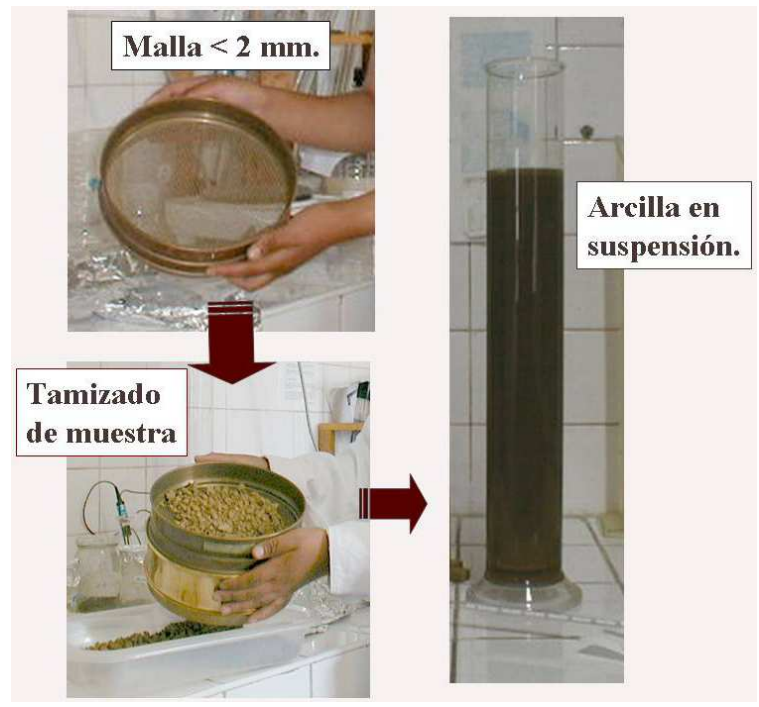

Figura 2: Obtención de la fracción arcillosa.

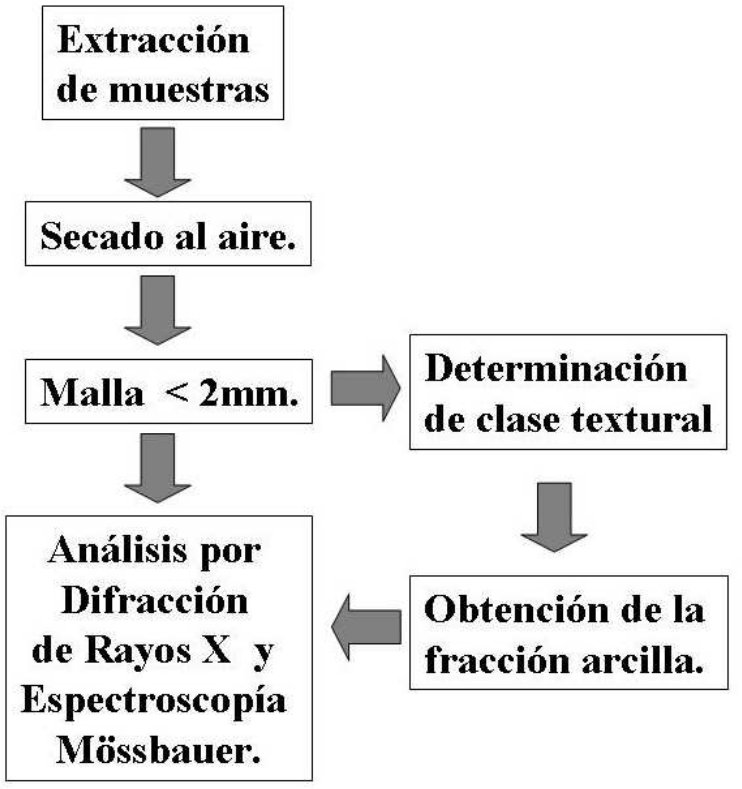

Figura 3: Protocolo de rutina para análisis de muestras.

\section{Espectroscopia Mössbauer}

El equipo empleado corresponde a un espectrómetro convencional de aceleración constante que se utilizó en la modalidad de transmisión. Se empleó una fuente radioactiva de $\mathrm{Co}^{57}$ en una matriz de Rhodio con actividad estimada de $5 \mathrm{mCi}$. Las medidas a temperatura de ambiente se llevaron a cabo en el Laboratorio de Arqueometría de la Facultad de Ciencias Físicas de la 
Universidad Nacional Mayor de San Marcos. Los espectros fueron ajustados haciendo uso del programa NORMOS [2]. La muestra fue colocada en un portamuestras circular de $1.7 \mathrm{~cm}$. de diámetro interior. Se empleo 220 mg de muestra en polvo para lograr un adecuado espesor óptico.

\section{Resultados y Discusión}

En la figura 4 se observa los resultados por la DRX de la muestra SI53 en bruto a $30 \mathrm{~cm}$ de profundidad, cuyas fases estructurales corresponden al cuarzo, contmorillonita, clinocloro, moscovita, anfibol y albita.

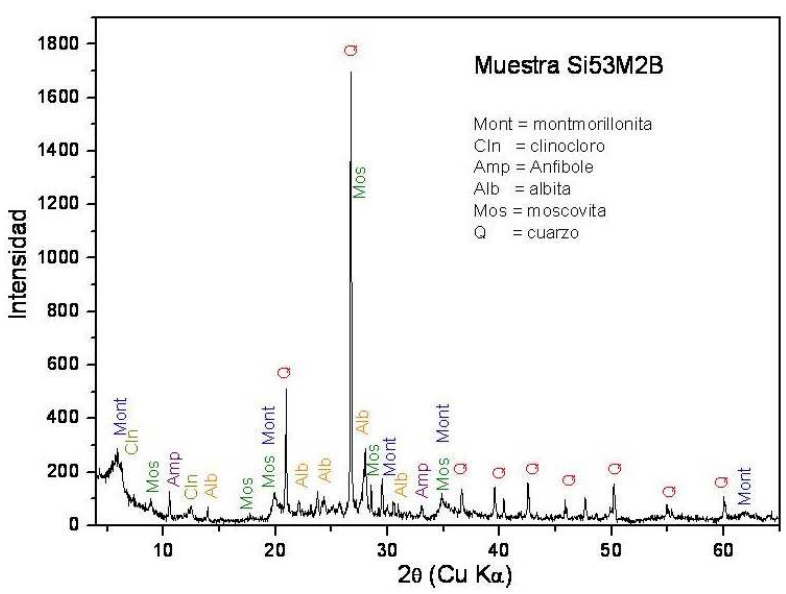

Figura 4: Difractograma de rayos X de la muestra SI53 en bruto a $30 \mathrm{~cm}$ de profundidad.

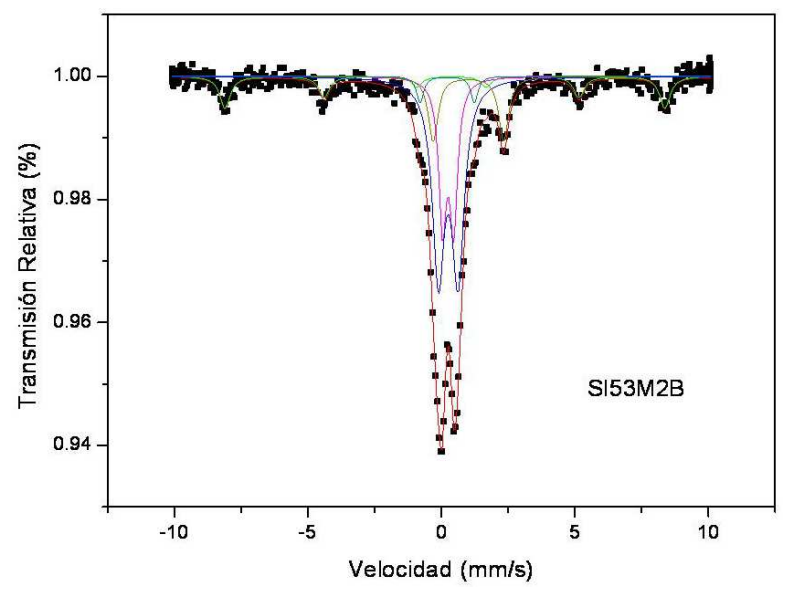

Figura 5: Espectro Mössbauer de la muestra SI53 en bruto a $30 \mathrm{~cm}$ de profundidad.

De acuerdo a los parámetros hiperfinos [3], en la figura 5 se observa, en el espectro Mössbauer tomado a temperatura ambiente, la presencia de un sexteto magnético adjudicado a la hematita y cuatro dobletes paramagnéticos de los cuales un doblete central puede ser adjudicado a algún óxido en estado superparamagnético o a la caolinita y los otros tres dobletes son adjudicados a la montmorillonita, la moscovita y la epidota.

En la figura 6 se observa los resultados por la DRX de la fracción arcillosa de la muestra SI53; se identifican las fases correspondientes al cuarzo, montmorillonita, clinocloro, moscovita y albita. En la tabla 1 se muestran las fichas ICCD de las muestras analizadas por difractometría de rayos X. En la figura 7, observamos el espectro Mössbauer de esta misma muestra, se identifica la presencia de un sexteto magnético correspondiente a la hematita y tres dobletes paramagnéticos de los cuales el doblete central puede ser adjudicado a algún óxido en estado superparamagnético como la caolinita, asimismo los otros dos dobletes son adjudicados a la montmorillonita y moscovita.

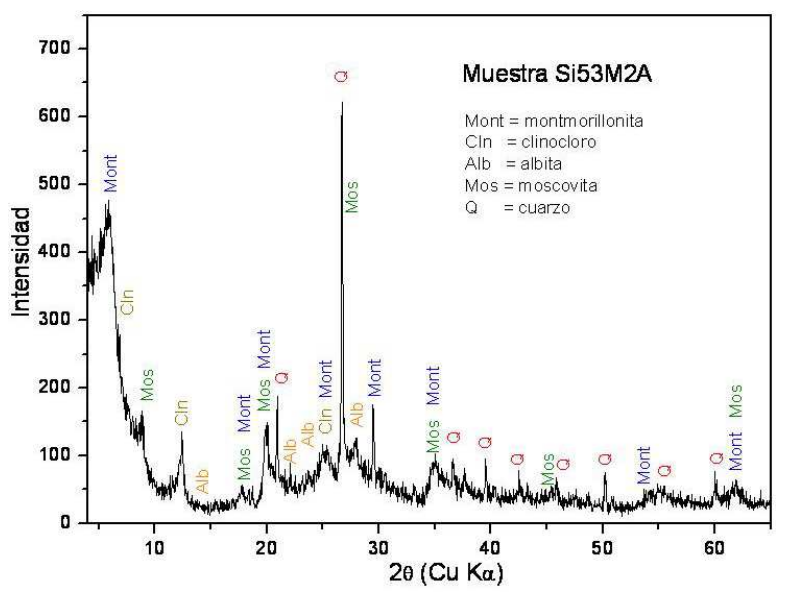

Figura 6: Difractograma de rayos X de la muestra SI53 en su fracción arcillosa que fue extraída a $30 \mathrm{~cm}$ de profundidad.

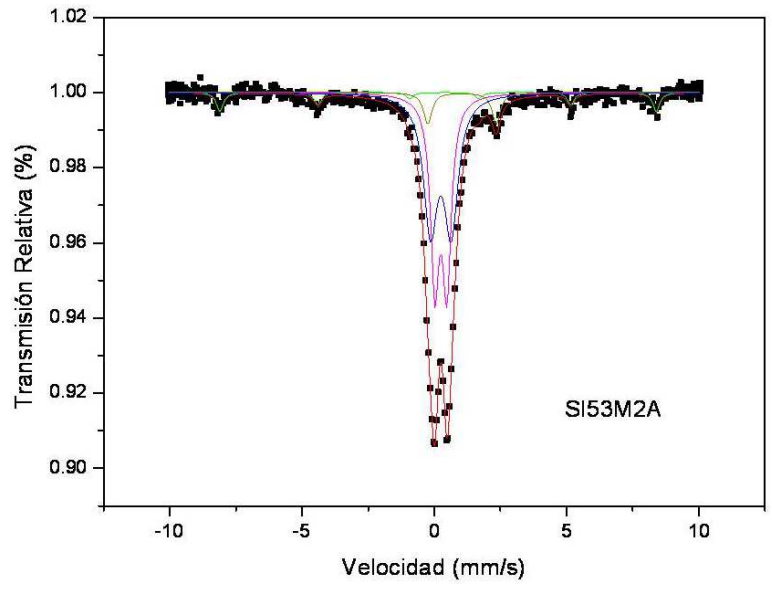

Figura 7: Espectro Mössbauer de la muestra SI53M2A. 
Los componentes mineralógicos que se observan en los espectros Mössbauer no son observados por la DRX debido a que se encuentran en estado amorfo o en una concentración relativamente muy baja. Asimismo, los componentes mineralógicos observados por la DRX y que no son observados en los espectros de la EMT se debe básicamente a que éstos no contienen hierro, por esta razón pasan desapercibidos por esta técnica.
En la tabla 2 se muestran los parámetros hiperfinos de las muestras a temperatura de ambiente 3. que se han obtenido y la tabla 3 muestra las áreas totales y parciales, asimismo el procentaje de la absorción resonante para cada uno de estos minerales. Estas áreas proporcionan una idea de la abundancia relativa del hierro presente en cada uno de estos minerales.

\begin{tabular}{lcc}
\cline { 1 - 1 } Muestras & SI53M2B & SI53M2A \\
\cline { 1 - 1 } Fases & & \\
\cline { 1 - 2 } Montmorillonite & {$[13-135]$} & {$[13-135]$} \\
Clinochlore & {$[29-701]$} & {$[29-701]$} \\
Muscovite & {$[7-42]$} & {$[7-42]$} \\
Amphibole & {$[73-1135]$} & \\
Quartz & {$[86-1628]$} & {$[86-1628]$} \\
Albite & {$[78-1995]$} & {$[78-1995]$} \\
\hline
\end{tabular}

Tabla 1: Fichas ICDD de las muestras analizadas por difractometría de rayos $\mathrm{X}$.

\begin{tabular}{lccc}
\hline Muestra & \multicolumn{3}{c}{ SI53M2B } \\
\cline { 2 - 4 } & ISO $(\mathrm{mm} / \mathrm{s}$ & QUA $(\mathrm{mm} / \mathrm{s})$ & BHF $(\mathrm{T})$ \\
\hline Hematita & 0.258 & -0.247 & 51.06 \\
Óxido superparamag- & 0.254 & 0.510 & \\
nético/Caolinita & & & \\
Montmorillonita & 0.252 & 0.741 & \\
Moscovita & 1.020 & 2.610 & \\
Epidota & 0.225 & 2.060 & \\
\hline Muestra & & SI53M2A & \\
\cline { 2 - 4 } & ISO $(\mathrm{mm} / \mathrm{s})$ & QUA $(\mathrm{mm} / \mathrm{s})$ & $\mathrm{BHF}(\mathrm{T})$ \\
\hline Hematita & 0.260 & -0.236 & 51.11 \\
Oxido superparamag- & 0.252 & 0.501 & \\
nético/Caolinita & & & \\
Montmorillonita & 0.249 & 0.709 & \\
Moscovita & 1.040 & 2.580 & \\
\hline Tabla 2: Parnetros & & & \\
\hline
\end{tabular}

Tabla 2: Parámetros hiperfinos de los minerales identificados en la parte bruta y en la fracción arcillosa.

\begin{tabular}{lllll}
\hline Muestra & \multicolumn{2}{c}{ SI53M2B } & \multicolumn{2}{c}{ SI53M2A } \\
\cline { 2 - 5 } & $\begin{array}{l}\text { Porcentaje de } \\
\text { absorción }(\%)\end{array}$ & $\begin{array}{l}\text { Área de absor- } \\
\text { ción }(\mathrm{mm} / \mathrm{s})\end{array}$ & $\begin{array}{l}\text { Porcentaje de } \\
\text { absorción }(\%)\end{array}$ & $\begin{array}{l}\text { Área de Absor- } \\
\text { ción }(\mathrm{mm} / \mathrm{s})\end{array}$ \\
\hline $\begin{array}{l}\text { Hematita } \\
\text { Óxido Superparamag- } \\
\text { nético/Caolinita }\end{array}$ & 13.437 & 0.015402 & 6.453 & 0.009738 \\
$\begin{array}{l}\text { Montmorillonita } \\
\text { Moscovita }\end{array}$ & 42.692 & 0.026009 & 40.765 & 0.061532 \\
Epidota & 12.679 & 0.054411 & 46.139 & 0.069632 \\
\hline Total & 3.7220 & 0.014533 & 6.6430 & 0.010025 \\
\hline
\end{tabular}

Tabla 3: Porcentajes de absorción y áreas de absorción resonante de las muestras estudiadas. 


\section{Conclusiones}

Los resultados obtenidos por la DRX para todas las muestras nos permiten identificar los minerales mayoritarios presentes que se encuentran aproximadamente en una proporción no menor al $5 \%$ y que no son amorfos; en la muestra en bruto se identificó la presencia de cuarzo, montmorillonita, clinocloro, moscovita, anfibol y albita; mientras que en la fracción arcillosa se identificó cuarzo, montmorillonita, clinocloro, moscovita y albita.

Por la EMT identificamos la presencia del óxido de hierro hematita, que pasa desapercibido a la difracción de rayos $\mathrm{X}$ por encontrarse en muy baja concentración. Existe un doblete que probablemente pertenezca a algún óxido en estado superparamagnético y que no se aprecia por la DRX debido a que se encontraría en el estado amorfo; los óxidos superparamagnéticos enmascaran su comportamiento magnético a temperatura ambiente [4]. Debido a que el efecto superparamagnético depende del tamaño de la partícula y de la constante de anisotropía, este efecto frecuentemente es exhibido por algunos óxidos con tamaño de grano menor de $10 \mathrm{~nm}$. La relajación superparamagnética puede ser impedida disminuyendo la temperatura [5]; por esto, la presencia de óxidos en estado superparamagnético se puede corroborar tomando el espectro a temperatura de nitrógeno o helio líquido. De no identificarse los óxidos en estado superparamagnético a bajas temperaturas, se concluiría que este doblete pertenecería a la caolinita. Por la EMT también se ha podido identificar sitios de hierro pertenecientes a minerales de arcilla como la moscovita y la montmorillonita. También se ha identificado la epidota.

Según los difractogramas de rayos $\mathrm{X}$ todas las especies minerales excepto el anfibol, se mantienen aún después del proceso de separación granulométrica por sedimentación. El espectro Mössbauer de la muestra correspondiente a la fracción arcilla nos permite afirmar que, salvo la epidota, todas las especies minerales se mantienen; sin embargo hay que indicar que en la fracción arcillosa la concentración de hematita ha disminuido, debido a que gran parte de ésta se ha sedimentado en el proceso de separación granulométrica. La epidota se ha sedimentado en su totalidad.

Para ambas muestras se puede establecer la complementariedad de las dos técnicas aplicadas pues los resultados por la EMT corroboran y complementan los resultados por la DRX, ya que ambos dan cuenta de la presencia de montmorillonita y moscovita.

\section{Comentarios finales}

Este trabajo constituye un estudio preliminar, parte de un trabajo de cooperación entre el Laboratorio de Análisis de Suelos de la Facultad de Ciencias Físicas de la Universidad Nacional Mayor de San Marcos y La Facultad de Agronomía de la Universidad Nacional Pedro Ruiz Gallo de Lambayeque; donde se espera poder concretar el estudio de las muestras pertenecientes a más sitios del Valle del Río Chancay y complementar además la información con otras técnicas analíticas como fluorescencia de rayos X para determinar la composición elemental y espectroscopia Mössbauer a bajas temperaturas.

Se espera que el presente trabajo sirva como fuente de información para el sector agrario de manera que se fortalezcan las aplicaciones de la ciencia del suelo y se incremente y armonice la comunicación con otras disciplinas. Con un conocimiento detallado de las propiedades del suelo y de su composición mineralógica y elemental, los profesionales del sector agrario tendrían mayor información para establecer mecanismos de manejo de suelos eficaces.

\section{Referencias}

[1] Manual de procedimientos de ensayos de Laboratorio, Facultad de Ciencias Físicas, UNMSM (2003).

[2] R. A. Brand, NORMOS Mössbauer Fitting Program, Olmos Tinajones (1997).

[3] J. Stevens, A. Kharanov, J. Miller, H. Pollac; Möss- bauer Mineral Handbook, Mössbauer Effect Data Center, Ashville, NC (1998).

[4] R. M. Cornell y U. Schwertmann; The Iron oxides, Structure, Properties, Reactions, Ocurrence and Uses, John Wiley \& Sons, New York (1997).

[5] Steen M $\phi$ rup y Elisabeth Tronc; Superparamagnetic relaxation of weakly interacting particles, Phys. Rev. Lett 72, 3278 (1994). 